\title{
Physiolibrary - Modelica library for Physiology
}

\author{
Marek Mateják*, Tomáš Kulhánek*, Jan Šilar*, Pavol Privitzer*, Filip Ježek**, Jiří Kofránek* \\ *Institute of Pathological Physiology, 1st Faculty of Medicine, Charles University in Prague \\ U nemocnice 5, Prague 2, 128 53, Czech Republic \\ **Department of Cybernetics, Faculty of Electrical Engineering, Czech Technical University in \\ Prague, Technicka 2, Prague 6 \\ marek@matfyz.cz
}

\begin{abstract}
Physiolibrary is a free open-source Modelica library designed for modeling human physiology. It is accessible on the Modelica Libraries web page at https://www.modelica.org/libraries. This library contains basic physical laws governing human physiology, usable for cardiovascular circulation, metabolic processes, nutrient distribution, thermoregulation, gases transport, electrolyte regulation, water distribution, hormonal regulation and pharmacological regulation.
\end{abstract}

Keywords: Physiolibrary; HumMod; Modelica library; Physiology; Integrative physiology; System biology

\section{Introduction}

Our laboratory have a long tradition building physiological libraries, starting with the Matlab/Simulink environment [2]. The origin of this Modelica Physiolibrary was in the first version of our HumMod Golem Edition model implementation [3-6], where it was called HumMod.Library. As the successors of Guyton's Medical Physiology School write, the original HumMod model [7] is "The best, most complete, mathematical model of human physiology ever created" [8].

We are also developing many types of smaller physiological models for use in medical education [9-11], so it was essential to separate this library from our HumMod Modelica implementation. Some other Modelica models and libraries covering the biological domain already existed, e.g. [12-17], which are useful in the process of system modeling and parameter identification. Especially BioChem Modelica library, that implements large part of SBML library in Modelica language [14-18].
Our Physiolibrary contains only carefully-chosen elementary physiological laws, which are the basis of more complex physiological processes. For example from only three type of blocks (ChemicalReaction, Substance and MolarConservationMass) it is possible to compose the allosteric transitions [19] or the Michaelis-Menten equation.

\section{Physiology}

Physiology is a very progressive discipline, that examines how the living body works. And it is no surprise that all processes in the human body are driven by physical laws of nature. The great challenge is to marry old empirical experiments with the "new" physical principles. Many teams and projects in the word deal with this formalization of physiology, for example: Physiome[20], SBML[17, 18], EuroPhysiome[21], VPH[22], CellML[23] etc. It is our hope that this library helps this unflagging effort of physiologists to exactly describe the processes.

\subsection{Display units in physiology}

Energy in medicine and chemistry has a very long tradition. One must not be confused by its different units and definitions. The researcher must be aware of multiple definitions of calorie, such as the international calorie, the $15^{\circ} \mathrm{C}$ calorie, the thermal calorie or the Calorie with a capital "C". The origin of this unit is in the thermal energy needed to heat one gram of water by one degree Celsius. But because the measurement conditions may differ, these alternative definitions are necessary. In physiology it is recommended to use only international calorie as defined in Table 1 . The flow of heat/energy is usually calculated in $\mathrm{kcal} / \mathrm{min}$, but in physics this is called power and is expressed in the SI unit watts.

Pressure units in medicine are also mainly based on historical measurements. For many years blood 
pressure was measured by the mercury sphygmomanometer, where the pressure is represented by the change of mercury hydrostatic column height. And because the scale of units on the column is in millimetres the pressure unit is called millimetre of mercury ' $\mathrm{mmHg}$ '. There also exists a very small difference between this unit and torrs. It is caused again by variance in measurement conditions.

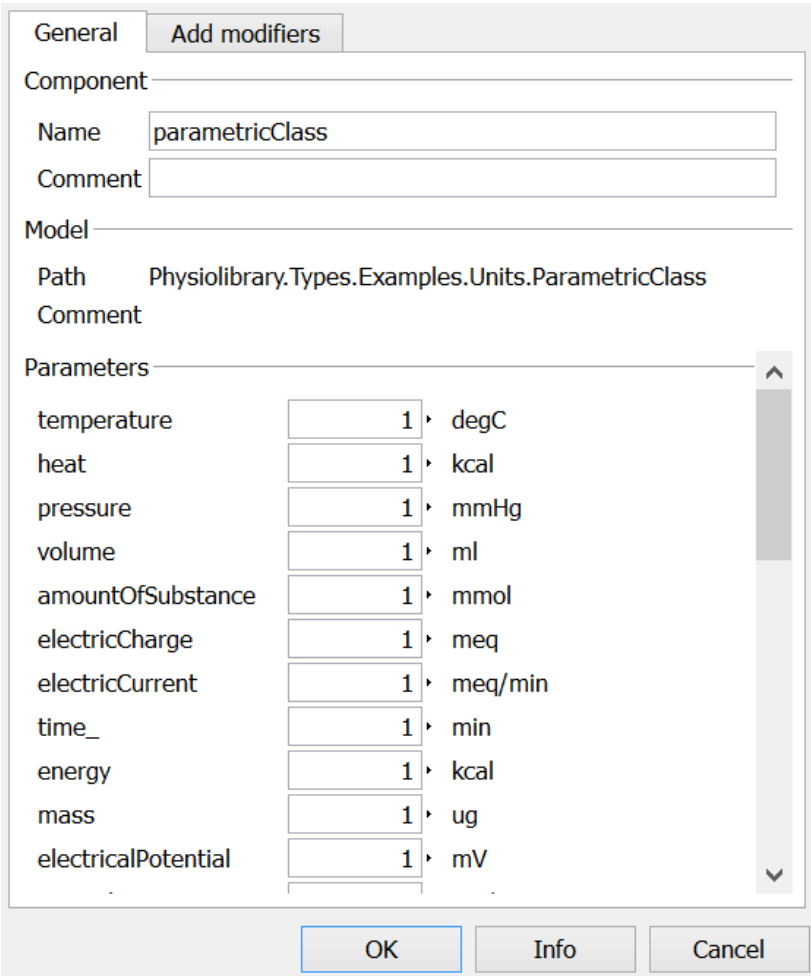

Figure 1, Example parameter dialog for non-SI physiological units. The Dymola environment automatically converts this user's non-SI-values to SI-values to ensure compatibility with any other Modelica library.

Many physiological processes are based on electrical principles in the human body. The main cause of this is that each cell has a nonconductive membrane with molecular structures called channels, through which the fluxes of electrolytes can be precisely regulated. Even more, the cells use energy from metabolism to retain a small electric potential between inside and outside. This view leads to a unit called equivalents or "eq". A charge of 1eq, for example, has $1 \mathrm{~mol}$ of sodium cations $\left(\mathrm{Na}^{+}\right)$. The fluxes of electrically charged ions can be in $\mathrm{meq} / \mathrm{min}$, but in physics the SI unit ampere is more generally used.

Unit conversion table

(for Modelica environment display-unit setting)

\begin{tabular}{llrl}
\hline \hline $\mathrm{x} \mathrm{kcal}$ & $=$ & $4186.8 * \mathrm{x}$ & $\mathrm{J}$ \\
\hline $\mathrm{x} \mathrm{kcal} / \mathrm{min}$ & $=$ & $69.78 * \mathrm{x}$ & $\mathrm{W}$ \\
\hline
\end{tabular}

\begin{tabular}{llrl}
\hline $\mathrm{x} \mathrm{mmHg}$ & $=$ & $133.322387415^{*} \mathrm{x}$ & $\mathrm{Pa}$ \\
\hline $\mathrm{x} \mathrm{degC}$ & $=$ & $273.15+\mathrm{x}$ & $\mathrm{K}$ \\
\hline $\mathrm{x} \mathrm{meq}$ & $=$ & $96.4853365 * \mathrm{x}$ & $\mathrm{C}$ \\
\hline $\mathrm{x} \mathrm{meq} / \mathrm{min}$ & $=$ & $1.60808894 * \mathrm{x}$ & $\mathrm{A}$ \\
\hline $\mathrm{x} \mathrm{mosm}$ & $=$ & $0.001 * \mathrm{x}$ & $\mathrm{mol}$ \\
\hline $\mathrm{x}$ litreSTP & $=$ & $0.044031617 * \mathrm{x}$ & $\mathrm{mol}$ \\
\hline $\mathrm{x}$ litreSATP & $=$ & $0.040339548 * \mathrm{x}$ & $\mathrm{mol}$ \\
\hline $\mathrm{x}$ litreNIST & $=$ & $0.041571200^{*} \mathrm{x}$ & $\mathrm{mol}$ \\
\hline \hline
\end{tabular}

Table 1, Selected Non-SI units in physiology

Another strange unit describing the amount of substance is the osmol ("osm"), which has the same value as the mol, but which highlights the property that this substance cannot cross the membrane together with the flux of its solvent.

For gases, it is common to measure the amount as volume, which for specific measurement conditions is equivalent to the number of molecules. The International Union of Pure and Applied Chemistry (IUPAC) set this standard condition for temperature and pressure (STP) precisely at $0^{\circ} \mathrm{C}$ and $100 \mathrm{kPa}$. But other standards exist. For example, SATP is measured at $25^{\circ} \mathrm{C}$ and $100 \mathrm{kPa}$, or the standard measurement condition at the National Institute of Standards and Technology (NIST), which is $20^{\circ} \mathrm{C}$ and $101.325 \mathrm{kPa}$.

\subsection{Chemical domains in physiology}

In physiology books, chapters about chemical substances are organized by their types. The main reason for this is that each substance in the human body is regulated in a different way. For example the regulation of sodium is different from the regulation of potassium, and from the regulation of glucose, and so on. This view leads to the idea of having separate models of each substance. The origin of different flows and regulations is the (cellular) membrane. Water and solutions can cross it in different directions at the same time. Crossings occur for different reasons: water is driven mostly by osmotic gradients, electrolytes are driven by charge to reach Donnan's equilibrium, and some solutes can even be actively transported against their concentration or electrical gradients. And all this is specifically driven from the higher levels by neural and hormonal responses.

In Physiolibrary flows and fluxes of solutes are supported mostly by the Chemical package. All parts inside this Physiolibrary.Chemical package use the connector ChemicalPort, which defines the molar concentration and molar flow/flux rate of one solute. This is the supporting infrastructure for modeling membrane diffusion, accumulations of substances, 
reversal chemical reactions, Henry's law of gas solubility, dilution with additional solvent flow, membrane reabsorption, chemical degradation and physiological clearance.

For usage examples, please open the Chemical.Examples package, where the chemical reaction shown in Fig. 2 is implemented, along with many other chemical processes.

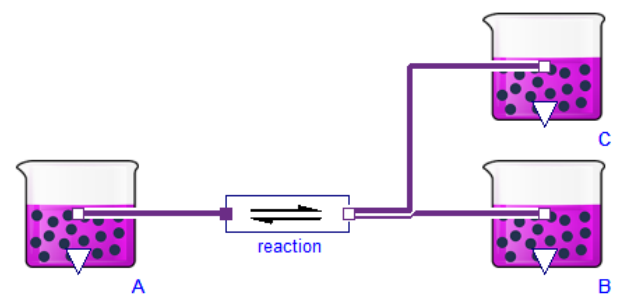

Figure 2, Example of a chemical reaction: A <-> B + C. Purple beakers (Substance) accumulate one type of substance and generate its concentration in port. Block for chemical reaction (ChemicalReaction) can have any number of substrates or products with any stoichiometric numbers. In this case there is only one substrate and two products. Purple lines represent chemical connectors, are composed of molar concentration and the molar flow of substance.

The graphically-created diagram shown in Fig. 2 generates this Modelica code:

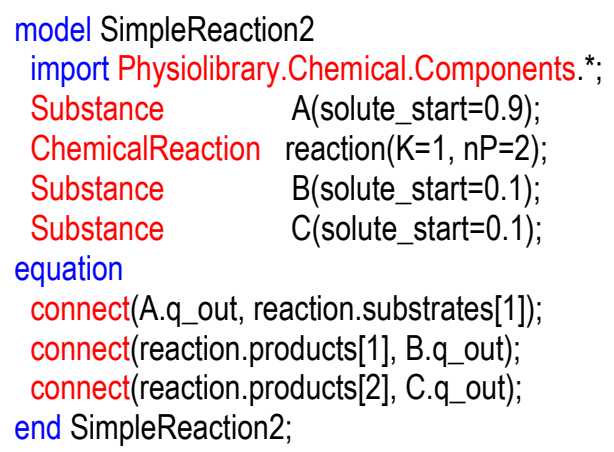

This means that before the numerical simulation begins, each Substance.solute_start parameter must be set to some initial amount of substance. ChemicalReaction.nP must also be configured for the number of products, and parameter ChemicalReaction.K must be configured for the dissociation constant of reaction in SI-units (please note that concentration of $1 \mathrm{~mol} / \mathrm{m}^{3}=1 \mathrm{mmol} / \mathrm{L}$ ). As mentioned before, the values in text code are in SIunits, but the Dymola environment support non-SI units in the parameter dialog of each component.

\subsection{Hydraulic domain in physiology}

The main usage of the hydraulic domain in human physiology is modeling of the cardio-vascular system. And because there are no extreme thermo- dynamic conditions, the system can be really simple - it is only necessary to model conditions for incompressible water, at normal liquid-water temperatures and with relative pressure $5-20 \mathrm{kPa}$. This boring thermodynamic state leads to using very simple blocks of hydraulic resistance, hydrostatic pressure, volumetric flow, inertia and finally a block representing blood accumulation in elastic vessels.

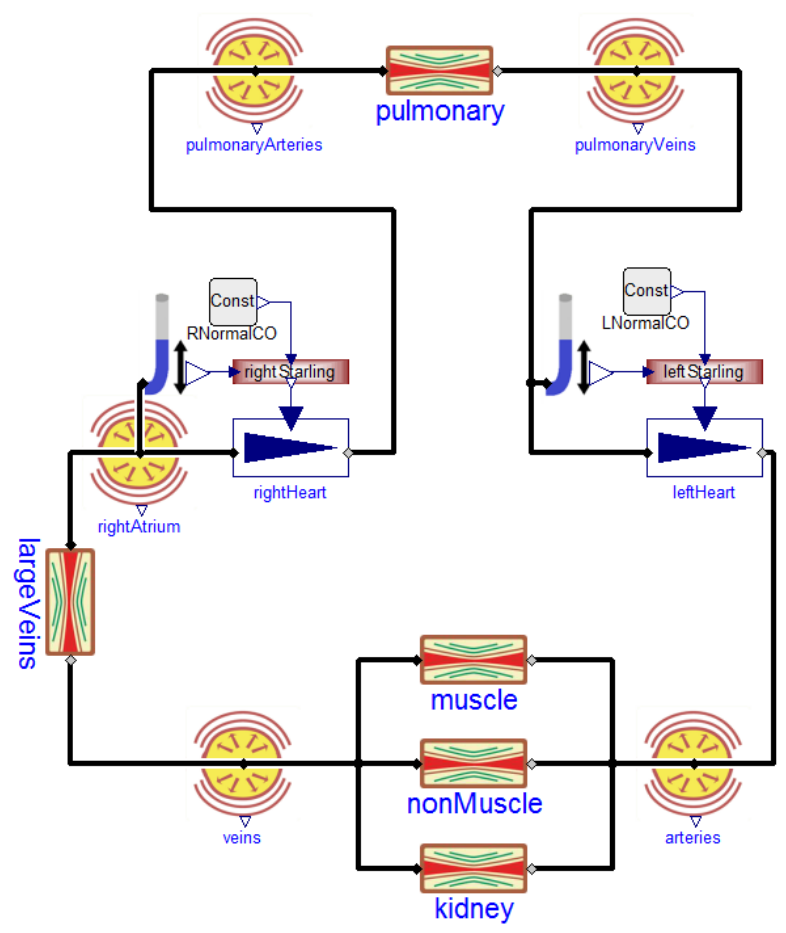

Figure 3, Hydraulic example: the cardiovascular subsystem of the famous Guyton-Coleman-Granger model [1]. Yellow circles (ElasticVessel) represent blood accumulation and pressure generation, rectangles between them are hydraulic resistances (Resistance) of blood vessels, blue triangles (Pump) represent a heart pump driven by the Frank-Starling law. Heart-filling pressures are determined by the block with the blue tube icon (PressureMeassure) and a block-rectangle (Blocks.Factors.Spline) converts filling pressure to their effect on cardiac output. Black lines connect the hydraulic connectors (HydraulicPort), which contains pressure and volumetric flow variables.

\subsection{Thermal domain in physiology}

For the human body to function optimally, it is critical to hold the core temperature at $35-39^{\circ} \mathrm{C}$. A fever of $41^{\circ} \mathrm{C}$ for more than a short period of time causes brain damage. If the core temperature falls below $10^{\circ} \mathrm{C}$, the heart stops. As in the hydraulic domain, the thermal domain is simplified to these conditions.

The Physiolibrary.Thermal package extends the package Modelica.Thermal.HeatTransfer from 
Modelica Standard Library 3.2 (MSL), where the connector is composed of temperature and heat flow. The main blocks in Physiolibrary.Thermal are: Conductor, IdealRadiator and HeatAccumulation. The heat conductor conducts the heat from the source, such us muscles or metabolically active tissue, to its surrounding. IdealRadiator delivers heat to tissues by blood circulation. HeatAccumulation plays a role in accumulating thermal energy in each tissue mass driven by its heat capacity. We recommend using this block instead of Modelica.Thermal.HeatTransfer .HeatCapacitor to allow the possibility of variable mass amounts, and to have support for calculating steady state, described in section 2.7 .

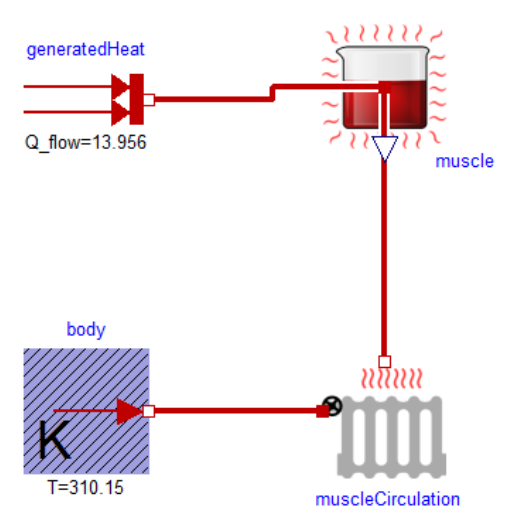

Figure 4, Example of heat flow from a working muscle. The muscle is represented by a red beaker (HeatAccumulation), where heat energy is accumulated in a mass with defined weight and specific heat. Heat transfer is processed by blood circulation (IdealRadiator) with blood flow as its internal parameter. The temperature of blood is set to a fixed value of $37^{\circ} \mathrm{C}$ to simulate well-regulated core body temperature.

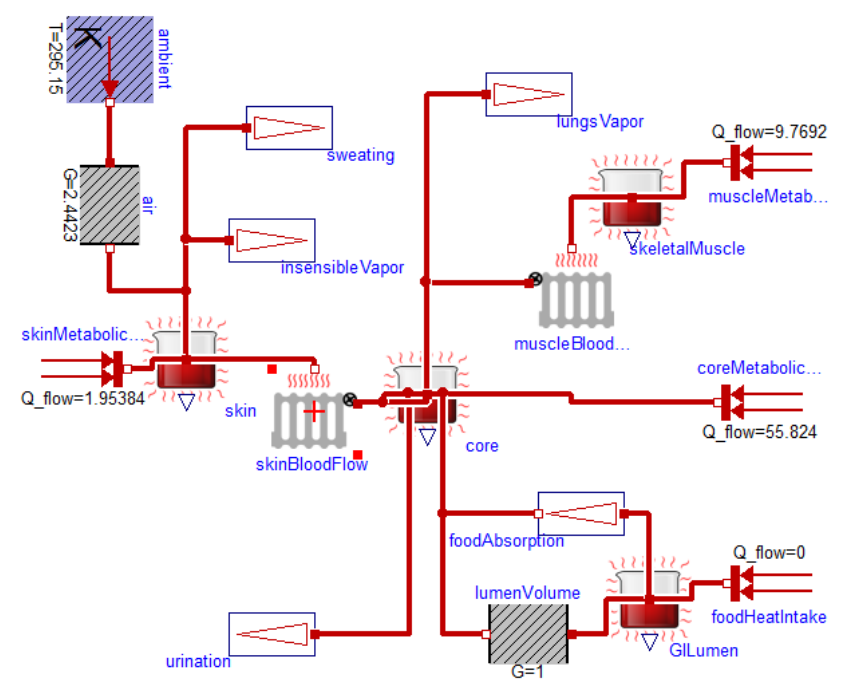

Figure 5, Basic heat flow model of human body. Heat production occurs in each tissue via metabolism, or from warm (to cool) eaten food using the MSL block with two red arrows (Modelica.Thermal.HeatTransfer. Sources.FixedHeatFlow). This heat is stored in tissues (HeatAccumulation) and transferred out by blood (IdealRadiator) or together with mass (Stream, HeatOutstream), where the model also integrates vaporization heat loss. Heat radiation and conduction to the environment is simplified using an MSL block for heat conductor (Modelica.Thermal.HeatTransfer. Components.ThermalConductor).

\subsection{Osmotic domain in physiology}

One of the basic phenomenon of biological systems is the osmotically-driven flow of water. This is always connected with semipermeable membranes. The different concentrations of impermeable solutes on both sides of the membrane causes the hydrostatic pressure at the concentrated side to rise[24]. This pressure difference is called osmotic pressure. Osmotic pressure is linearly proportional to the concentration gradient of impermeable solutes. The osmolarity (osmotic concentration) is also one of the main indexes of human body balance, called homeostasis. Its value should not significantly deviate for a long period of time from a value of $285-295 \mathrm{mosm} / 1$.

In Physiolibrary the osmotic connector OsmoticPort is composed of the osmotic concentration and the volumetric flux of permeable liquid. The two main blocks are called Membrane and OsmoticCell. Here, inside the membrane blocks, it is of course possible to also define hydraulic pressure and temperatures effects on both sides of membrane.

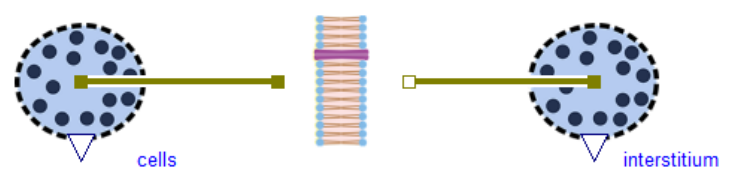

Figure 6, Osmotic example simulating water transfer between intracellular and interstitial compartments in hypertonic or hypotonic conditions.

\subsection{Types and units}

The most common errors in HumMod Golem Edition were caused by using bad physical units. The main problem of medical research, articles, and experiments is using obscure units from medicine, pharmacology, biology and non-physics disciplines.

The Physiolibrary fulfills the Modelica ideal of using SI units as the main unit for each variable, and the previously described physiological units are also implemented as the displayUnits for each variable. Using these displayUnits the user sets and sees the "physiological" values. The implementation can also be joined to any unit-correct Modelica models and physical equations without crashing due to unit in- 
compatibilities. The unit support of Physiolibrary is so strong that one can even chose the right unit-typed "input real" $/$ "output real" from the library package Types.RealIO or one can use unit-typed constants (Types.Constants). As can be expected, only the nonspecific package Blocks in the Physiolibrary has variables without units.

\subsection{Steady states}

One of the main questions in clinical medicine is how to stabilize the patient. In modeling the oscillating heart, breathing, circadian rhythm or menstruation cycle the model can be designed as nonoscillating, using variables such as period times, amplitudes, frequencies, mean values and other phase space variables. This type of model has better numerical stability for long simulation times, and even more importantly, it can be "stabilized". This stabilization we call a steady state.

To be mathematically exact, we define a steady state system (SSS) as a non-differential system derived from an original differential system (DS) by using zero derivations and by adding additional steady state equations (ASSE). The number of the ASSE must be the same as the number of algebraically dependent equations in the non-differential system derived from DS by setting zero derivations. The ASSE describes the system mostly from the top view, such as the equations of mass conservation laws or the boundary equation of environment sources.

To define a model as an SSS, the user must switch each Simulation parameter in each block to the value Types.SimulationType.SteadyState and must have correctly defined all necessary ASSE. This setting causes the system to ignore any start values for any state and add zero derivation equations instead. There does not currently exist a Modelica environment which can automatically find and remove generated dependent equations by this solution. So the correct number of states must be marked as dependent (parameter isDependent) and the same number of ASSE must be inserted. However, despite the fact that a model in this steady-state setting will not be locally balanced, it must be globally balanced and without any dependent equation.

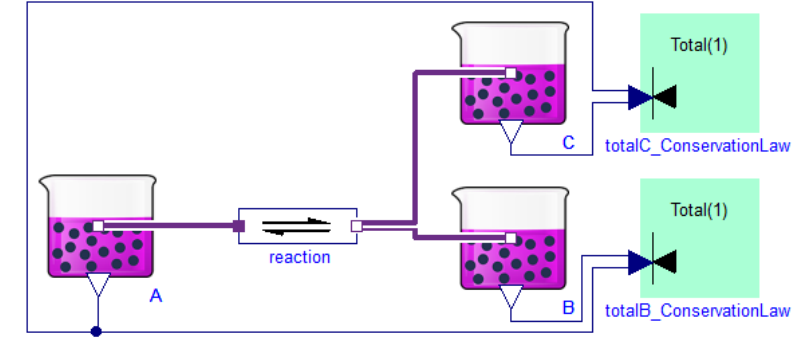

Figure 7, Steady state system example: equilibrium of chemical reaction $\mathrm{A}<->\mathrm{B}+\mathrm{C}$ is calculated with two ASSE. Zero change of reactant $A$ is automatically propagated through a reaction to both products. So both products must be marked as dependent (parameter isDependent), and two mass conservation laws must be added as green square blocks to the right (MolarConservationLaw). Please note that conservation laws must be included only after designing the rest of the system, because they are global properties, not properties of individual substances or reactions.

Adding an ASSE is possible by inserting and connecting the energy or mass conservation law block from package SteadyState.Components. Another possibility is using environment sources blocks, where setting the isIsolatedInSteadyState parameter adds the equation of the zero mass/volume/energy flow from or to environment.

The steady state model often changes to one big nonlinear strong component, but without solver stiff or convergence problems. Especially in quick chemical kinetics, it is not necessary to have very rapid molar fluxes, when it always reach equilibrium in the end. This design is also useful in creating steadystated parts in a dynamical model without huge rebuilding. It also brings other benefits. To see these possibilities, one has to realize that conservation laws could be invariances in a dynamical simulation. This is really useful for debugging.

For example see the model SteadyStates.Examples.SimpleReaction2_in_Equilib rium (Fig.7), which implements the equilibrium of the closed system from Fig.2 as a solution of three chemical substances with a simple reversible reaction between them extended by two conservation laws. Each of these laws describes the total possible amount of one product in its free form and in its associated form.

It is always a big challenge to nicely solve initial values of differential system. However, it should be possible to solve the SSS in its initial phase. And this is the idea behind the Types.SimulationType.InitSteadyState option for models already extended with ASSE. 
[2] Teaching, L.o.B.a.C.A. Physiolibrary in Matlab and Simuling. 2008; Available from: http://www.physiome.cz/simchips.

[3] Marek Mateják and J. Kofránek, HUMMODGOLEM EDITION-ROZSÁHLÝ MODEL FYZIOLOGICKÝCH SYSTÉM ${ }^{\circ}$ Medsoft, 2011: p. 182-196.

[4] Marek Mateják and J. Kofránek, Rozsáhlý model fyziologických regulací v Modelice. Medsoft, 2010: p. 126-146.

[5] Kofránek, J., M. Mateják, and P. Privitzer. HumMod - large scale physiological model in Modelica. in 8th. International Modelica conference. 2011. Dresden, Germany.

[6] Kofránek, J., Mateják, M., Privitzer, P., Tribula, M., Kulhánek, T., Šilar, J., Pecinovský, R. HumMod-Golem Edition: large scale model of integrative physiology for virtual patient simulators. in World Congress in Computer Science 2013 (WORLDCOMP'13), International Conference on Modeling, Simulation and Visualisation Methods (MSV'13). 2013.

[7] Hester, R.L., et al., HumMod: a modeling environment for the simulation of integrative human physiology. Frontiers in physiology, 2011. 2.

[8] Center, L.b.U.o.M.M., HumMod. 2012: p. http://hummod.org/.

[9] Kofránek, J., M. Mateják, and P. Privitzer. Leaving toil to machines - building simulation kernel of educational software in modern software environments. in Mefanet 2009. 2009. Masaryk University, Brno.

[10] Kofránek, J., M. Mateják, and P. Privitzer, Web simulator creation technology. MEFANET report, 2010. 3: p. 52-97.

[11] Kofranek, J., et al., The Atlas of Physiology and Pathophysiology: Web-based multimedia enabled interactive simulations. Computer methods and programs in biomedicine, 2011. 104(2): p. 143153.

[12] Proß, S. and B. Bachmann, An Advanced Environment for Hybrid Modeling and Parameter Identification of Biological Systems.

[13] Cellier, F.E. and A. Nebot. Object-oriented Modeling in the Service of Medicine. in Proc. 6th Asia Simulation Conference. 2005.

[14] Larsdotter Nilsson, E. and P. Fritzson. BioChemA Biological and Chemical Library for Modelica. in Proceedings of the 3rd International Modelica Conference (November 3-4, Linköping, Sweden). 2003. Modelica Assocation.

[15] Nilsson, E.L. and P. Fritzson, Biochemical and metabolic modeling and simulation with Modelica. BioMedSim. Linköping, Sweden, 2005.

[16] Nilsson, E.L. and P. Fritzson. A Metabolic Specialization of a General Purpose Modelica Library for Biological and Biochemical Systems. in Paper presented at the 4th International Modelica Conference. 2005.

[17] Brugård, J., et al. Creating a Bridge between Modelica and the Systems Biology Community. in 7th International Modelica Conference, Como, Italy. 2009.

[18] Hucka, M., et al., The systems biology markup language (SBML): a medium for representation and exchange of biochemical network models. Bioinformatics, 2003. 19(4): p. 524-531.

[19] Monod, J., J. Wyman, and J.-P. Changeux, On the nature of allosteric transitions: a plausible model. Journal of Molecular Biology, 1965. 12(1): p. 88-118.

[20] Bassingthwaighte, J.B., Strategies for the physiome project. Annals of Biomedical Engineering, 2000. 28(8): p. 1043-1058.

[21] Fenner, J.W., et al., The EuroPhysiome, STEP and a roadmap for the virtual physiological human. Philosophical Transactions of the Royal Society A: Mathematical, Physical and Engineering Sciences, 2008. 366(1878): p. 29792999.

[22] Hunter, P.J. and M. Viceconti, The VPHphysiome project: standards and tools for multiscale modeling in clinical applications. Biomedical Engineering, IEEE Reviews in, 2009. 2: p. 40-53.

[23] Smith, L., et al., SBML and CellML translation in Antimony and JSim. Bioinformatics, 2013: p. btt641.

[24] Mortimer, R.G., Mathematics for physical chemistry. 1999: Access Online via Elsevier. 